\title{
Integral elastic, vibrational-excitation, electronic-state excitation, ionization, and total cross sections for electron scattering from para-benzoquinone
}

Cite as: J. Chem. Phys. 148, 204305 (2018); https://doi.org/10.1063/1.5028298

Submitted: 08 March 2018. Accepted: 09 May 2018. Published Online: 30 May 2018

D. B. Jones (D, R. F. da Costa, F. Kossoski (D), M. T. do N. Varella, M. H. F. Bettega (D), C. García, F. Blanco (D), R. D. White, M. A. P. Lima (D), and M. J. Brunger
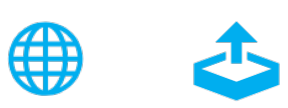

\section{ARTICLES YOU MAY BE INTERESTED IN}

Electron-impact electronic-state excitation of para-benzoquinone

The Journal of Chemical Physics 148, 124312 (2018); https://doi.org/10.1063/1.5023494

Elastic scattering and vibrational excitation for electron impact on para-benzoquinone

The Journal of Chemical Physics 147, 244304 (2017); https://doi.org/10.1063/1.5010831

An ab initio investigation for elastic and electronically inelastic electron scattering from para-benzoquinone

The Journal of Chemical Physics 149, 174308 (2018); https://doi.org/10.1063/1.5050622
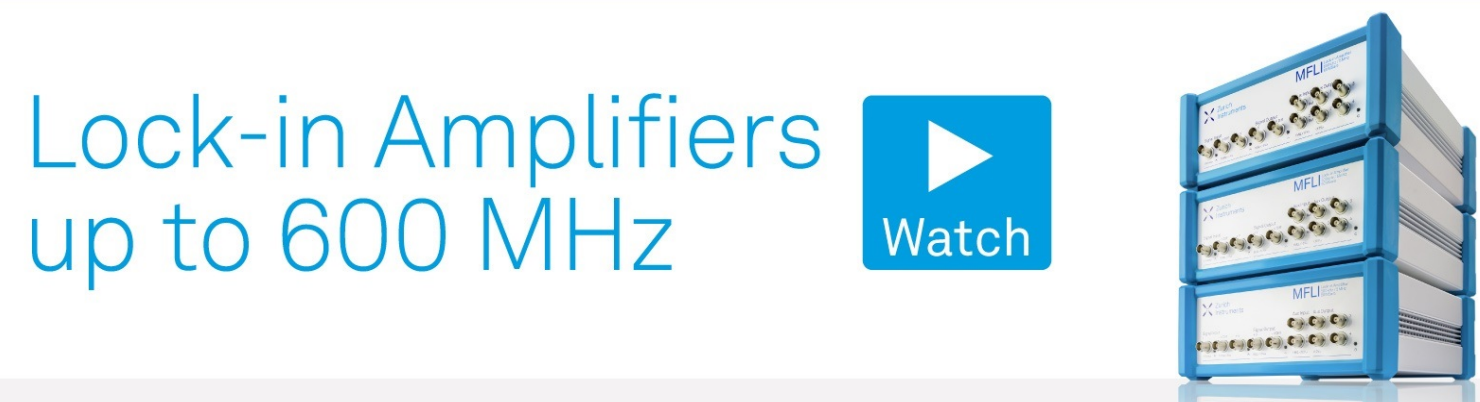


\title{
Integral elastic, vibrational-excitation, electronic-state excitation, ionization, and total cross sections for electron scattering from para-benzoquinone
}

\author{
D. B. Jones, ${ }^{1}$ R. F. da Costa, ${ }^{2,3}$ F. Kossoski, ${ }^{2}$ M. T. do N. Varella, ${ }^{4}$ M. H. F. Bettega, ${ }^{5}$ \\ G. García, ${ }^{6}$ F. Blanco, ${ }^{7}$ R. D. White, ${ }^{8}$ M. A. P. Lima, ${ }^{2, a)}$ and M. J. Brunger ${ }^{1, a)}$ \\ ${ }^{1}$ College of Science and Engineering, Flinders University, GPO Box 2100, Adelaide, SA 5001, Australia \\ ${ }^{2}$ Instituto de Física "Gleb Wataghin," Universidade Estadual de Campinas, 13083-859 Campinas, \\ São Paulo, Brazil \\ ${ }^{3}$ Centro de Ciências Exatas, Departamento de Física, Universidade Federal do Espírito Santo, \\ 29075-901 Vitória, Espírito Santo, Brazil \\ ${ }^{4}$ Instituto de Física, Universidade de São Paulo, Rua do Matão 1731, 05508-090 São Paulo, São Paulo, Brazil \\ ${ }^{5}$ Departamento de Física, Universidade Federal do Paraná, CP 19044, 81531-990 Curitiba, Paraná, Brazil \\ ${ }^{6}$ Instituto de Física Fundamental, CSIC, Serrano 113-bis, E-28006 Madrid, Spain \\ ${ }^{7}$ Departamento de Física Atómica, Molecular y Nuclear, Universidad Complutense de Madrid, \\ Madrid E-28040, Spain \\ ${ }^{8}$ College of Science and Engineering, James Cook University, Townsville, Qld 4811, Australia
}

(Received 8 March 2018; accepted 9 May 2018; published online 30 May 2018)

\begin{abstract}
We report absolute experimental integral cross sections (ICSs) for the electron impact excitation of 6 bands (Bands 0-V) of unresolved electronic-states in para-benzoquinone, for incident electron energies between 20 and $40 \mathrm{eV}$. Absolute vibrational-excitation ICSs, for 3 composite vibrational bands (Bands I-III), are also reported in that same energy range. In addition, ICSs calculated within our independent atom model (IAM) with screening corrected additivity rule (SCAR) formalism, extended to account for interference (I) terms that arise due to the multi-centre nature of the scattering problem, are also reported. The sum of those ICSs gives the IAM-SCAR+I total cross section (TCS) for electron-para-benzoquinone scattering. Where possible, those calculated IAM-SCAR+I ICSs are compared against corresponding results from the present measurements with an acceptable level of accord being obtained. Similarly, we also present results from our Schwinger multichannel method with pseudopotential (SMCPP) calculations. Here elastic ICSs and ICSs corresponding to the Bands 0-III of unresolved electronic-states are presented, with agreement between the SMCPP electronic-state ICSs and those from our measurements being in good qualitative accord. The energy range of our SMCPP computations is 16-50 eV. Using the binary-encounter-Bethe (BEB) approach, total ionization cross sections for this collision system were computed. Those total ionization cross sections were then added to our SMCPP ICS results, to derive SMCPP/BEB TCSs that are typically in very good accord with those from our IAM-SCAR+I approach. Published by AIP Publishing. https://doi.org/10.1063/1.5028298
\end{abstract}

\section{INTRODUCTION}

In recent times, there has been quite a deal of interest in simulating charged-particle tracks in gases and liquids (see, e.g., Refs. 1-6), and in particular for biomolecules that might help us to understand radiation damage in matter at the nanoscale. There has also been renewed interest, specifically in considering the behavior of electron swarms under the influence of an applied electric field, as they traverse a background gas or liquid of biomolecules (see, e.g., Refs. 7-10). In addition, significant work has been undertaken by the lowtemperature plasma communities in modeling the behavior of their plasmas in such applications as medicine, ${ }^{11}$ converting biomass into useful "green" fuels, ${ }^{12}$ and in the more traditional area of materials processing for technological devices. ${ }^{13}$

a) Authors to whom correspondence should be addressed: michael.brunger@ flinders.edu.au and maplima@ifi.unicamp.br
Similarly, the study of atmospheric plasma behavior remains a very active area of scientific endeavour. ${ }^{14-18}$ Underpinning at least some aspects of the simulations currently being conducted is the need for what Tanaka et al. ${ }^{19}$ described as having electron scattering cross section databases that were "correct, absolute, and comprehensive." To that end, various communities have worked very hard to assemble extensive databases with two relatively recent examples of this being the Virtual Atomic and Molecular Data Center ${ }^{20}$ and $\mathrm{LXCaT}^{21}$ More specialised database reviews from Brunger and Buckman, ${ }^{22}$ Yoon et al. ${ }^{23}$ Anzai et al. ${ }^{24}$ Brunger $^{25}$ and Gorfinkiel and Ptasinska ${ }^{26}$ should also be noted.

We have recently ${ }^{27-29}$ described why benzoquinones are important in biological systems, through their ability to undergo reversible chemical reduction as a key mechanism within photosynthesis or cellular respiration, and that quinone derivatives possibly represent sustainable materials for use in energy harvesting and storage. ${ }^{30,31}$ As a consequence, the 
simplest quinone, namely, para-benzoquinone (pBQ), serves as an ideal prototypical structure for better understanding electron transport in quinone chemistry. Aside from our own contribution $^{27-29}$ to establishing a fundamental database, in order to be able to undertake Monte Carlo or Boltzmann equation transport simulations, in electron-pBQ scattering, we also note some earlier (non-absolute) work from Allan ${ }^{32,33}$ and a quite detailed R-matrix investigation from Loupas and Gorfinkiel. ${ }^{34}$ Clearly, therefore, there is still much work to be done in $\mathrm{pBQ}$ $\left(\mathrm{C}_{6} \mathrm{H}_{4} \mathrm{O}_{2}\right)$ to meet the requirement outlined above by Tanaka et al. ${ }^{19}$ with the current contribution seeking to further extend the available absolute cross section database for electron-pBQ scattering.

The structure of the remainder of this paper is as follows. In Sec. II, we give details of our analysis procedures and theory. As such details have already been given recently, ${ }^{35,36}$ we will be relatively brief here. Thereafter, in Sec. III, our results and a discussion of those results are presented, with some conclusions from this investigation finally being proffered in Sec. IV.

\section{ANALYSIS PROCEDURES AND THEORY}

All details of the vibrational quanta of $\mathrm{pBQ}$ can be found in the work of Jones et al., ${ }^{28}$ while the relevant excited electronic-state spectroscopy of $\mathrm{pBQ}$ is given in the work of Jones et $a .^{27,29}$ As a consequence, we do not repeat all that information again here. Rather, we simply note that experimental integral cross sections (ICSs) for the (i) Band I vibrational quanta (unresolved CC-stretching/CHbending/CO stretching modes), (ii) Band II vibrational quanta (unresolved CH-stretching modes), (iii) Band III vibrational quanta (unresolved combination bands), (iv) Band 0 excited electronic-states, (v) Band I excited electronic-states, (vi) Band II excited electronic-states, (vii) Band III excited electronic-states, (viii) Band IV excited electronic-states, and (ix) Band V excited electronic-states are reported in this paper. The sum of the ICS for those 3 "discrete" vibrational bands and 6 "discrete" electronic-state bands is also reported. The energy range for the present experimental ICS is $20-40 \mathrm{eV}$, with a listing of the vibrational ICS being given in Table I and the electronic-state ICS being found in Table II. Plots of those data can be seen in Figs. 1 and 2, respectively.

A comprehensive description of our experimental and data analysis methodologies, in going from measured electron energy loss spectra to deriving the absolute inelastic differential cross sections (DCSs), can be found in the work of Jones et al. $^{27-29}$ (to whom the reader is referred for more details). The DCS $\left(\sigma_{j}\right)$ for a given scattering process, $j$ [in this case, $j=$ (i)-(ix) above], is related to the ICS, $Q_{j}$, through the

TABLE I. Experimental integral cross sections $\left(\times 10^{-16} \mathrm{~cm}^{2}\right)$ for the electron impact vibrational excitation of pBQ. Note that $\mathrm{E}_{\mathrm{L}}$ denotes the energy loss.

\begin{tabular}{|c|c|c|c|c|c|c|c|c|}
\hline \multirow{3}{*}{$\begin{array}{l}\text { Energy } \\
(\mathrm{eV})\end{array}$} & \multicolumn{2}{|c|}{ Vib. Band I } & \multicolumn{2}{|c|}{ Vib. Band II } & \multicolumn{2}{|c|}{ Vib. Band III } & \multicolumn{2}{|c|}{ Vib. sum } \\
\hline & \multicolumn{2}{|c|}{$\left(\mathrm{E}_{\mathrm{L}} \sim 0.11-0.21 \mathrm{eV}\right)$} & \multicolumn{2}{|c|}{$\left(\mathrm{E}_{\mathrm{L}} \sim 0.37 \mathrm{eV}\right)$} & \multicolumn{2}{|c|}{$\left(\mathrm{E}_{\mathrm{L}} \sim 0.53 \mathrm{eV}\right)$} & \multirow[b]{2}{*}{$Q_{\text {sum }}$} & \multirow[b]{2}{*}{ Abs. error } \\
\hline & $Q_{\mathrm{i}}$ & Abs. error & $Q_{\mathrm{ii}}$ & Abs. error & $Q_{\mathrm{iii}}$ & Abs. error & & \\
\hline 20 & 2.09 & 1.64 & 0.219 & 0.108 & 0.030 & 0.019 & 2.34 & 1.64 \\
\hline 30 & 1.50 & 1.23 & 0.101 & 0.057 & 0.012 & 0.011 & 1.61 & 1.23 \\
\hline 40 & 1.53 & 1.15 & 0.091 & 0.059 & 0.014 & 0.012 & 1.64 & 1.15 \\
\hline
\end{tabular}

TABLE II. Experimental integral cross sections $\left(\times 10^{-16} \mathrm{~cm}^{2}\right)$ for the electron impact electronic-state excitation of $\mathrm{pBQ}$.

\begin{tabular}{|c|c|c|c|c|c|c|}
\hline \multirow{2}{*}{$\begin{array}{l}\text { Energy } \\
(\mathrm{eV})\end{array}$} & \multicolumn{2}{|c|}{ Band $0\left(\mathrm{E}_{\mathrm{L}} \sim 3.0 \mathrm{eV}\right)$} & \multicolumn{2}{|c|}{ Band I $\left(\mathrm{E}_{\mathrm{L}} \sim 4.3 \mathrm{eV}\right)$} & \multicolumn{2}{|c|}{ Band II $\left(\mathrm{E}_{\mathrm{L}} \sim 5.5 \mathrm{eV}\right)$} \\
\hline & $Q_{\mathrm{iv}}$ & Abs. error & $\mathrm{Q}_{\mathrm{v}}$ & Abs. error & $Q_{\mathrm{vi}}$ & Abs. error \\
\hline 20 & 0.20 & 0.12 & 0.26 & 0.14 & 1.52 & 0.60 \\
\hline 30 & 0.18 & 0.13 & 0.30 & 0.20 & 1.88 & 0.69 \\
\hline 40 & 0.08 & 0.07 & 0.07 & 0.05 & 1.58 & 0.40 \\
\hline \multirow{2}{*}{$\begin{array}{l}\text { Energy } \\
(\mathrm{eV})\end{array}$} & \multicolumn{2}{|c|}{ Band III $\left(\mathrm{E}_{\mathrm{L}} \sim 6.4 \mathrm{eV}\right)$} & \multicolumn{2}{|c|}{ Band IV $\left(\mathrm{E}_{\mathrm{L}} \sim 7.8 \mathrm{eV}\right)$} & \multicolumn{2}{|c|}{ Band V $\left(\mathrm{E}_{\mathrm{L}} \sim 9.6 \mathrm{eV}\right)$} \\
\hline & $Q_{\text {vii }}$ & Abs. error & $Q_{\text {viii }}$ & Abs. error & $Q_{\text {ix }}$ & Abs. error \\
\hline 20 & 0.39 & 0.24 & 1.71 & 0.72 & 1.63 & 0.94 \\
\hline 30 & 0.48 & 0.30 & 2.54 & 1.19 & 2.18 & 1.18 \\
\hline 40 & 0.10 & 0.07 & 1.69 & 0.56 & 0.95 & 0.46 \\
\hline \multirow{2}{*}{$\begin{array}{l}\text { Energy } \\
(\mathrm{eV})\end{array}$} & \multicolumn{2}{|c|}{ Electronic sum } & & & & \\
\hline & $Q_{\text {sum }}$ & Abs. error & & & & \\
\hline 20 & 5.70 & 1.36 & & & & \\
\hline 30 & 7.57 & 1.86 & & & & \\
\hline 40 & 4.46 & 0.83 & & & & \\
\hline
\end{tabular}




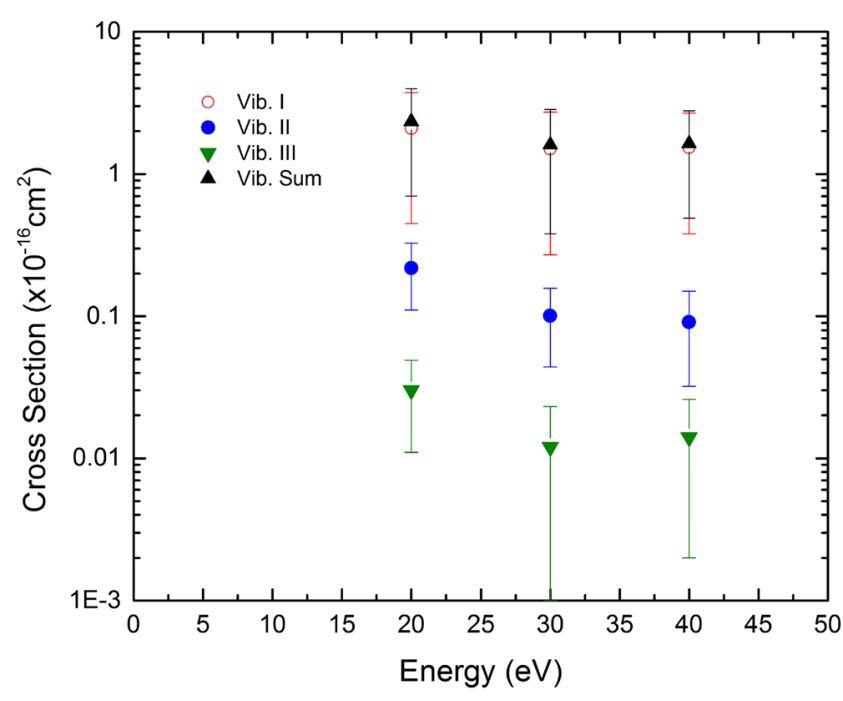

FIG. 1. Experimental ICS $\left(\times 10^{-16} \mathrm{~cm}^{2}\right)$ for the electron impact excitation of composite vibrational bands, and the summed vibrational bands, in $\mathrm{pBQ}$. See legend in figure and text for further details.

standard formula

$$
Q_{j}\left(E_{0}\right)=2 \pi \int_{0}^{\pi} \sigma_{j}\left(E_{0}, \theta\right) \sin \theta d \theta,
$$

where $E_{0}=$ incident electron energy and $\theta=$ scattered electron angle. In order to convert experimental DCS data, measured at specific angles which span a finite angular range determined by the physical constraints of the apparatus, ${ }^{37}$ to an ICS, one must first extrapolate and interpolate the measured data so that they cover the full angular range from $0^{\circ}$ to $180^{\circ}$. Our approach to accomplish this has also been described in some detail previously, ${ }^{19}$ and so again, we do not repeat that detail here. We do however note that for optically allowed transitions our extrapolation is based around a generalised oscillator strength (GOS) formalism ${ }^{38,39}$ to account for the strongly forward peaked behavior of the DCS. The absolute uncertainties, which depend on the $E_{0}$ and inelastic channel in question, on the present derived ICSs are also given in Tables I and II and

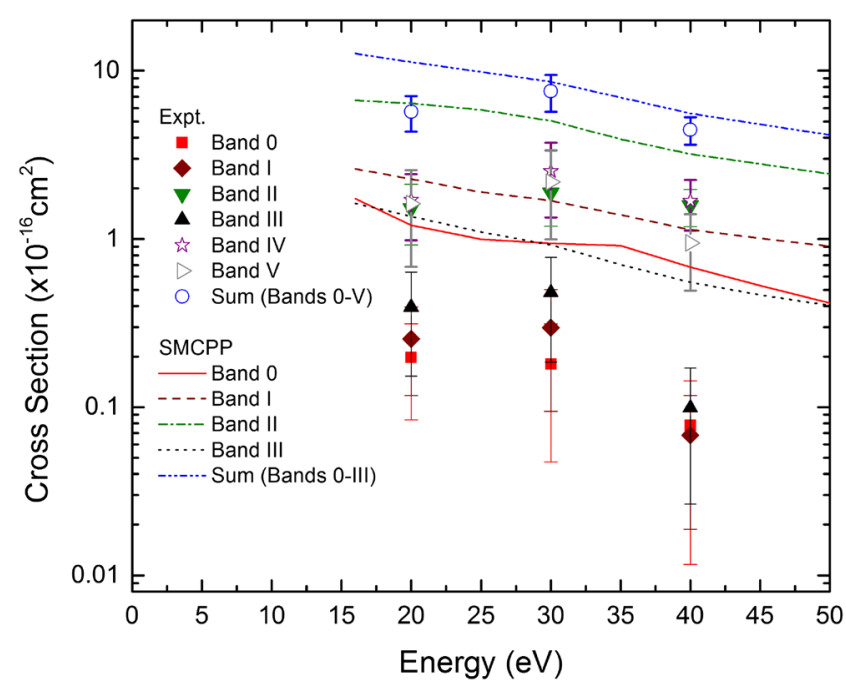

FIG. 2. Present experimental and theoretical SMCPP ICS $\left(\times 10^{-16} \mathrm{~cm}^{2}\right)$ for the electron impact excitation of composite electronic-state bands, and the summation of those electronic-state bands, in pBQ. Please refer to legend and text for further details. are all at the one standard deviation level. Those uncertainties arise from the intrinsic errors on the measured DCS and any uncertainty introduced through the interpolation/extrapolation of our data.

We have discussed our independent atom modelscreening corrected additivity rule (IAM-SCAR) computations on numerous occasions ${ }^{40-42}$ so that only a précis needs to be given here. In essence, an atomic optical potential scattering model calculates all the phase shifts for each of the atomic species found within the molecule in question (i.e., carbon, oxygen, and hydrogen for $\mathrm{pBQ}$ ). The molecular scattering amplitudes then stem from the sum of all the relevant atomic amplitudes, including the phase coefficients. This is the additivity rule (AR). However the AR does not account for the target molecular structure so that some screening coefficients (SCs) are also employed in order to account for the geometry of the molecule (atomic positions and bond lengths). Additionally, Blanco and García ${ }^{43}$ have introduced an interference term (I) to help us describe that the collision dynamics involve scattering from multiple centres. In general, the IAM-SCAR and/or IAM-SCAR+I approaches have shown an efficacy for describing electron-atom and electron-molecule collisions down to $\sim 20 \mathrm{eV}$ (sometimes even lower); e.g., refer to Refs. 44-46, and later, we will examine its applicability to the present electron-pBQ scattering system. In Table III, we list all the relevant IAM-SCAR+I cross section data from the current calculations, with plots of those ICSs and the total cross section (TCS) being found in Fig. 3. Note that in Tables III and IV, we also include total ionization cross sections (TICSs) from our binary-encounter-Bethe (BEB) computations. ${ }^{19,47}$ In the BEB approximation, the integral ionization cross section contributions from the $i$ th molecular orbital, $Q_{i}$, is obtained via

$$
Q_{i}\left(t_{i}\right)=\frac{4 \pi a_{0}^{2} N_{i}\left(R / B_{i}\right)^{2}}{t_{i}+u_{i}+1}\left[\frac{\ln \left(t_{i}\right)}{2}\left(1-\frac{1}{t_{i}^{2}}\right)+1-\frac{1}{t_{i}}-\frac{\ln \left(t_{i}\right)}{t_{i}+1}\right] .
$$

In Eq. (2), $t_{i}=E_{0} / B_{i}$ and $u_{i}=U_{i} / B_{i}$, with $a_{0}$ and $R$ being the Bohr radius and Rydberg energy, respectively. $N_{i}, B_{i}$, and $U_{i}$ are the ionized orbital's occupation number, bound-state binding energy, and average orbital kinetic energy, respectively. At Flinders University, the pBQ average orbital kinetic energies and binding energies were obtained using a hybrid OVGF/B3LYP with aug-cc-pVDZ quantum chemical description. Note that the acronym OVGF represents outer valence Green function. At the Federal University of Paraná, an independent BEB calculation using a HF/aug-cc-pVDZ quantum chemical model, at the same geometry used in the scattering calculations with the SMCPP method, was also performed. The total ionization cross section, $Q_{\text {ion }}$ or TICS, see Tables III and IV, is then obtained in each case by summing up over the cross section contributions from the $N$-occupied pBQ orbitals,

$$
Q_{\text {ion }}\left(E_{0}\right)=\sum_{i=1}^{N} Q_{i}\left(t_{i}\right) \text {. }
$$

The HF ionization energies are unphysical, and this shifts the maximum in the BEB cross section to higher impact energies, with a lower magnitude of the TICS. 
TABLE III. The present IAM-SCAR+I cross section set for electron scattering from pBQ. The TICS in this table is calculated at the BEB level using a hybrid OVGF/B3LYP quantum chemical description. ${ }^{*}$ The electronic excitation cross section is obtained by subtracting the BEB TICS from the IAM-SCAR+I total inelastic cross section.

\begin{tabular}{|c|c|c|c|c|c|}
\hline $\begin{array}{l}\text { Energy } \\
(\mathrm{eV})\end{array}$ & $\begin{array}{c}\text { Elastic } \\
\left(\times 10^{-16} \mathrm{~cm}^{2}\right)\end{array}$ & $\begin{array}{c}\text { Inelastic } \\
\left(\times 10^{-16} \mathrm{~cm}^{2}\right)\end{array}$ & $\begin{array}{c}\text { TCS } \\
\left(\times 10^{-16} \mathrm{~cm}^{2}\right)\end{array}$ & $\begin{array}{l}\text { TICS (BEB) } \\
\left(\times 10^{-16} \mathrm{~cm}^{2}\right)\end{array}$ & $\begin{array}{l}\text { Electronic* } \\
\left(\times 10^{-16} \mathrm{~cm}^{2}\right)\end{array}$ \\
\hline 0.1 & 131.04 & 0 & 131.04 & & \\
\hline 0.15 & 144.76 & 0 & 144.76 & & \\
\hline 0.2 & 151.76 & 0 & 151.76 & & \\
\hline 0.3 & 155.68 & 0 & 155.68 & & \\
\hline 0.4 & 152.32 & 0 & 152.32 & & \\
\hline 0.5 & 143.64 & 0 & 143.64 & & \\
\hline 0.7 & 124.32 & 0 & 124.32 & & \\
\hline 1 & 106.12 & 0 & 106.12 & & \\
\hline 1.5 & 88.76 & 0 & 88.76 & & \\
\hline 2 & 79.80 & 0 & 79.80 & & \\
\hline 3 & 71.40 & 0 & 71.40 & & \\
\hline 4 & 66.92 & 0 & 66.92 & & \\
\hline 5 & 64.12 & 0 & 64.12 & & \\
\hline 7 & 61.32 & 0 & 61.32 & & 0.00 \\
\hline 10 & 59.08 & 0.14 & 59.36 & 0.00 & 0.14 \\
\hline 15 & 54.60 & 3.64 & 58.24 & 1.58 & 2.06 \\
\hline 20 & 48.72 & 9.04 & 57.68 & 4.51 & 4.53 \\
\hline 30 & 42.00 & 15.04 & 57.12 & 9.36 & 5.68 \\
\hline 40 & 37.80 & 16.86 & 54.88 & 12.23 & 4.63 \\
\hline 50 & 34.72 & 17.42 & 52.08 & 13.83 & 3.59 \\
\hline 70 & 30.24 & 17.39 & 47.60 & 15.08 & 2.31 \\
\hline 100 & 26.18 & 16.52 & 42.56 & 15.05 & 1.47 \\
\hline 150 & 21.90 & 14.78 & 36.68 & 13.78 & 1.00 \\
\hline 200 & 19.10 & 13.33 & 32.48 & 12.39 & 0.94 \\
\hline 300 & 15.43 & 11.17 & 26.60 & 10.17 & 1.00 \\
\hline 400 & 13.08 & 9.63 & 22.71 & 8.61 & 1.02 \\
\hline 500 & 11.40 & 8.48 & 19.91 & 7.47 & 1.01 \\
\hline 700 & 9.13 & 6.89 & 16.04 & 5.94 & 0.95 \\
\hline 1000 & 7.08 & 5.43 & 12.52 & 4.57 & 0.86 \\
\hline
\end{tabular}

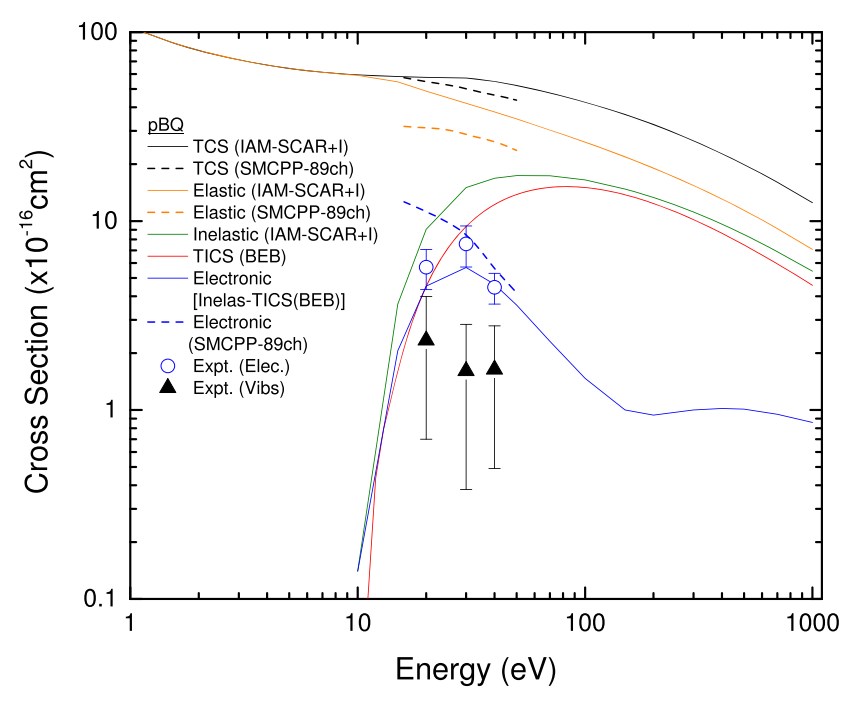

FIG. 3. Experimental and theoretical cross sections $\left(\times 10^{-16} \mathrm{~cm}^{2}\right)$ for electron scattering from $\mathrm{pBQ}$ and for various scattering processes. The SMCPP total cross section is the sum of the elastic ICS, the summation of electronic-state band ICSs, and the TICS calculated at the BEB level, as implemented with a HF/aug-cc-pVDZ quantum chemical description. See legend in figure and text for further details.
The Schwinger multichannel method (SMC) $)^{48}$ is a variational approach that uses square integrable basis functions to obtain the scattering amplitude for electron collisions with molecules of arbitrary geometry. The method takes into account important effects such as the electron exchange, the electrontarget polarization interaction, and, also, the possibility of flux competition between the elastic and inelastic channels through electronic multichannel coupling. In the present study, we used the parallel version ${ }^{49}$ of the SMC implementation that employs norm-conserving pseudopotentials (SMCPP) ${ }^{50}$ and single-excitation configuration interaction techniques for the target description by means of the minimal orbital basis for single configuration interaction (MOBSCI) strategy. ${ }^{51}$ Since the method and its computational implementation were recently reviewed, ${ }^{52}$ here we only give the working expression for the scattering amplitude

$$
f\left(\mathbf{k}_{f}, \mathbf{k}_{i}\right)=-\frac{1}{2 \pi} \sum_{m, n}\left\langle S_{\mathbf{k}_{f}}|V| \chi_{m}\right\rangle\left(d^{-1}\right)_{m n}\left\langle\chi_{n}|V| S_{\mathbf{k}_{i}}\right\rangle,
$$

where 
TABLE IV. Summary of our SMCPP results for integral cross sections and for elastic and discrete inelastic processes, when electrons interact with pBQ. The Bands 0-III are the ICS for the composite electronic states, while the Sum 0-III is the electronic sum. Here we also include the ICS contribution from electronic state excitation to the remaining energetically open states (other electronic) that cannot be reasonably assigned to specific excitation processes within the MOBSCI framework. The TICS is obtained at the BEB level using a HF/aug-cc-pVDZ quantum chemical model. The TCS is obtained by summing up the elastic ICS, the electronic ICS (for all energetically open channels), and the TICS.

\begin{tabular}{|c|c|c|c|c|c|c|c|c|c|}
\hline $\begin{array}{l}\text { Energy } \\
(\mathrm{eV})\end{array}$ & $\begin{array}{c}\text { Elastic } \\
\left(\times 10^{-16} \mathrm{~cm}^{2}\right)\end{array}$ & $\begin{array}{c}\text { Band 0 } \\
\left(\times 10^{-16} \mathrm{~cm}^{2}\right)\end{array}$ & $\begin{array}{c}\text { Band I } \\
\left(\times 10^{-16} \mathrm{~cm}^{2}\right)\end{array}$ & $\begin{array}{c}\text { Band II } \\
\left(\times 10^{-16} \mathrm{~cm}^{2}\right)\end{array}$ & $\begin{array}{c}\text { Band III } \\
\left(\times 10^{-16} \mathrm{~cm}^{2}\right)\end{array}$ & $\begin{array}{c}\text { Electronic } \\
\text { Sum }(0-\mathrm{III}) \\
\left(\times 10^{-16} \mathrm{~cm}^{2}\right)\end{array}$ & $\begin{array}{c}\text { Other } \\
\text { Electronic } \\
\left(\times 10^{-16} \mathrm{~cm}^{2}\right)\end{array}$ & $\begin{array}{c}\text { TICS } \\
\left(\times 10^{-16} \mathrm{~cm}^{2}\right)\end{array}$ & $\begin{array}{c}\text { TCS } \\
\left(\times 10^{-16} \mathrm{~cm}^{2}\right)\end{array}$ \\
\hline 16 & 31.71 & 1.74 & 2.61 & 6.66 & 1.63 & 12.63 & 11.66 & 1.36 & 57.36 \\
\hline 20 & 31.10 & 1.21 & 2.28 & 6.40 & 1.36 & 11.25 & 9.04 & 3.13 & 54.52 \\
\hline 25 & 30.62 & 1.00 & 1.90 & 5.85 & 1.10 & 9.85 & 6.93 & 5.37 & 52.76 \\
\hline 30 & 28.73 & 0.94 & 1.69 & 5.04 & 0.92 & 8.60 & 5.66 & 7.20 & 50.19 \\
\hline 35 & 27.44 & 0.91 & 1.39 & 3.93 & 0.71 & 6.94 & 4.87 & 8.64 & 47.89 \\
\hline 40 & 26.33 & 0.68 & 1.14 & 3.20 & 0.55 & 5.57 & 4.73 & 9.75 & 46.39 \\
\hline 45 & 25.01 & 0.53 & 1.01 & 2.79 & 0.47 & 4.80 & 4.63 & 10.61 & 45.04 \\
\hline 50 & 23.65 & 0.42 & 0.91 & 2.44 & 0.40 & 4.16 & 4.57 & 11.25 & 43.63 \\
\hline
\end{tabular}

$$
d_{m n}=\left\langle\chi_{m}\left|\left[\frac{\hat{H}}{N+1}-\frac{\hat{H} P+P \hat{H}}{2}+\frac{P V+V P}{2}-V G_{P}^{(+)} V\right]\right| \chi_{n}\right\rangle .
$$

In Eqs. (4) and (5), $P$ is a projector onto $N_{\text {open }}$ energy-allowed target electronic channels, i.e.,

$$
P=\sum_{\ell=1}^{N_{\text {open }}}\left|\Phi_{\ell}\right\rangle\left\langle\Phi_{\ell}\right|,
$$

$G_{P}^{(+)}$is the free-particle Green's function projected onto the $P$ space, $V$ is the interaction potential between the electron and the molecular target, $\mathbf{k}_{i}\left(\mathbf{k}_{f}\right)$ is the incoming (outgoing) electron wave vector, and $\hat{H}=E-H$ is the total collision energy minus the Hamiltonian of the $(N+1)$-electron system under the fixed nuclei approximation. $H$ is defined as $H=H_{0}+V$, where $H_{0}$ represents the Hamiltonian for the non-interacting electron-molecule system and $S_{\mathbf{k}}$ is its corresponding solution, given by the product of a plane wave (projectile) and a target state $\Phi_{\ell}$. For the expansion of the variational scattering wave function, the method employs trial basis sets comprising $(N+1)$-particle configuration state functions (CSFs), denoted by $\chi_{m}$, that are built from spin-adapted, anti-symmetrized products of target electronic-states and projectile scattering orbitals. The open electronic collision channels are included in the $P$ space, and the dynamical response of the target electrons to the projectile field (correlation-polarization effects) is accounted for through virtual excitations of the target. In this case, the CSFs are given by

$$
\left|\chi_{m}\right\rangle=\mathcal{A}_{N+1}\left|\Phi_{i}(1, \ldots, N)\right\rangle \otimes\left|\varphi_{j}(N+1)\right\rangle,
$$

where $\left|\Phi_{i}(1, \ldots, N)\right\rangle$ is a target state and $\left|\varphi_{j}(N+1)\right\rangle$ is a single-particle orbital which represents a scattering orbital.

The integral cross section for an electronic-state excitation process $\Phi_{n} \rightarrow \Phi_{n^{\prime}}$, where $\Phi_{n\left(n^{\prime}\right)}$ denotes the target states, can be readily obtained from the scattering amplitude given in Eq. (4) by the following expression:

$$
\sigma_{n \rightarrow n^{\prime}}(E)=\frac{k_{f}}{k_{i}} \frac{1}{4 \pi} \int d \hat{\mathbf{k}}_{i} \int d \hat{\mathbf{k}}_{f}\left|f\left(\mathbf{k}_{f}, \mathbf{k}_{i}\right)\right|^{2} .
$$

In Eq. (8), the magnitude of the final wave vector is given by $k_{f}^{2}=k_{i}^{2}-2\left(\epsilon_{n}^{\prime}-\epsilon_{n}\right)$, where $\epsilon_{n}$ denotes the energy of the $n$th electronic target state. Note that in this equation, atomic units are employed so that $\hbar=1$. The integration over $\hat{\mathbf{k}}_{f}$ accounts for scattering into all the possible directions, while $(4 \pi)^{-1} \int d \vec{k}_{i}$ averages over the random molecular orientations in the target gas. The integration over both the incoming and outgoing directions makes the ICS rotationally invariant and hence equal in both the molecule-fixed and laboratory-fixed reference frames. Finally, it is important to note that the ICSs for the electronic-state bands considered in this work (Bands $0-$ III) are simply obtained as a sum of the ICSs for each electronic excitation process involving the states that compose the specific band under consideration. All the present SMCPP ICS results, for Bands $0-$ III of unresolved excited electronic-states, are summarised in Table IV.

\section{RESULTS AND DISCUSSION}

Considering now Fig. 1, which plots all our experimental composite vibrational-mode ICSs (see also Table I), then it should be manifested that there are no other experimental measurements or theoretical calculations against which we can compare the current results. We do not underestimate the difficulty for theorists in moving away from a fixed nuclei framework, in order to calculate vibrational excitation cross sections. Nonetheless, various frameworks that enable vibrational quanta to be calculated do exist ${ }^{53-56}$ and have been quite successful in reproducing corresponding measured data. However, those earlier calculations have largely concentrated on homonuclear diatomics and polyatomics that are much smaller and have fewer electrons than $\mathrm{pBQ}$ does. Notwithstanding the above observations, it would be highly desirable if independent vibrational-excitation measurements and/or some scattering computations were to be undertaken on $\mathrm{pBQ}$. It is also clear from Fig. 1 that the ICS energy dependencies, for each of the bands of unresolved modes, are very similar to within the errors quoted. We also found that typically the ICS $\mathrm{Iband} \mathrm{I}_{\text {b }} \mathrm{ICS}_{\text {band II }}$

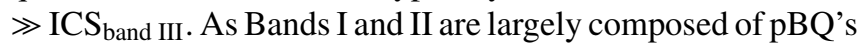
fundamental normal modes, whereas Band III is made up of combination bands (see Ref. 28), this observation, in regards to the ICS magnitudes, is not surprising and is consistent with what Jones et al. ${ }^{28}$ found at the differential cross section level. It is well known from infrared (IR) spectroscopy that the 
intensities of the fundamental modes of a molecule are greater than those for their overtone and combination lines. Basically, this is the same behavior that we are observing here for the Band III ICSs relative to those for Bands I and II (see Fig. 1). In the case of the magnitude of the Band I ICSs being significantly larger than those for Band II, given that both of these composite bands are composed of fundamental stretching and bending (Band I only) modes, there is in fact no contradiction. This follows as Band I consists of 19 vibrational quanta, while Band II consists of only 4 vibrational quanta (see Table II in Ref. 28). Thus the difference between their magnitudes is we believe largely due to a density of states argument, whereby as Band I incorporates about 5 times as many of the modes as Band II, it follows that one might reasonably expect that its ICSs would be larger. Again, as you would hope, all these observations in respect to the ICS magnitudes of the Bands IIII vibrational quanta are entirely consistent with those noted previously ${ }^{28}$ at the DCS level.

In Fig. 2 and Table II, we present our experimental ICSs for electron impact $\left(E_{0}=20-40 \mathrm{eV}\right)$ excitation of the Band 0 , Band I, Band II, Band III, Band IV, and Band V excited electronic-states in $\mathrm{pBQ}$. The spectroscopy of those bands of electronic-states was considered in detail previously in the work of Jones et al. ${ }^{27,29}$ to whom the reader is referred for more details. Also included in Fig. 2 are the corresponding ICSs we calculated using the SMCPP method, which employed 89-open electronic-states described within a MOBSCI framework. ${ }^{29}$ Note, however, that the SMCPP computations were restricted to the elastic channel and the Band 0, Band I, Band II, and Band III electronic-states, and further note that all our SMCPP results are converged by inclusion of partial waves up to $L=13$. A summary of the present SMCPP calculation results, for $E_{0}$ in the range $16-50 \mathrm{eV}$, within a static exchange plus polarisation (SEP) framework, ${ }^{29}$ can be found in Table IV. Considering Fig. 2 in more detail, then for each of the Bands 0, I, II, and III, we find a very good qualitative accord, to within the experimental uncertainties, between our measured ICSs and the SMCPP-SEP calculated ICSs in respect to their observed energy dependence. A discrepancy, however, between the magnitude of the ICSs, for each band of the theory and experiment, is observed with the theory always overestimating that found in the measurements. We believe the nature of this discrepancy, consistent with that found in our earlier phenol ${ }^{35}$ and furfural ${ }^{36}$ investigations, is due to the finite size of our present MOBSCI and, perhaps more significantly here as our MOBSCI is actually quite large, the fact that coupling to the continuum is currently not considered. Our earlier studies on the effect of multichannel coupling on the relevant cross section magnitudes clearly demonstrated its important role (see, e.g., Ref. 40). Therefore, we are currently trying to implement a strategy, admittedly a not fully ab initio strategy, by which ionization, as calculated by the BEB approach, is allowed to compete for flux with our SMCPP computation for the open channels within that calculation. At the very least, we anticipate that this approach should allow us to quantify in part the effect of the continuum on the elastic and discreteinelastic (electronic-state) channels, and our hope is that the discrepancy we observe between our theory and experimental ICS in Fig. 2 will be much less significant.
In Fig. 3 and Table III, we present our current IAMSCAR+I results for the integral elastic cross section, the total inelastic integral cross section (i.e., the sum of the ICSs for all the electronic-states, neutral dissociation, and the total ionization cross section), and the total cross section. By subtracting our BEB total ionization cross section (see also Table III), calculated using a hybrid OVGF/B3LYP quantum chemical description, we could obtain an estimate for the IAM-SCAR+I ICS for the sum of all the discrete electronic-states and neutral dissociation. This latter ICS is also listed in Table III under the column "Electronic" and plotted in Fig. 3. Also plotted in Fig. 3 are our SMCPP elastic ICS, sum over the electronic-state ICS for Bands 0-III, and a TCS formed from the preceding two ICSs and a BEB result, here however calculated with a HF/aug-cc-pVDZ quantum chemistry, for the total ionization cross section. All these latter SMCPP and BEB results can be found in Table IV. Finally, Fig. 3 additionally shows the experimental results for the sum of the ICSs for all the composite vibrational quanta (see Table I) and the sum of the ICS for all the electronic-state bands (see Table II). When we compared our summed vibrational ICS and summed electronic-state ICS to the TICS and elastic ICS from theory, we see that between 20 and $40 \mathrm{eV}$ they are much smaller in magnitude (particularly with respect to the elastic ICS). However as Brunger et al. ${ }^{5}$ recently demonstrated, just because a cross section is relatively small does not mean you can simply ignore it when undertaking simulations of charged-particle track behavior. Indeed they specifically found ${ }^{5}$ that in the final nano-volumes of the electron tracks in pyrimidine, the relatively small vibrational excitations become very important for a quantitative description of the system to be achieved. It is also interesting to compare in Fig. 3 the experimental summed electronic-state ICSs to the corresponding IAM-SCAR+I sum, although that latter work in principle also has a contribution from the neutral dissociation ICS. The very good level of agreement observed here strongly suggests that in the $20-40 \mathrm{eV}$ range the ICS for neutral dissociation in electron-pBQ scattering is likely very small. Finally, over most of the common energy range, we also note the very good qualitative level of accord between both our IAM-SCAR+I and SMCPP elastic ICSs, an observation consistent with what we previously discussed at the elastic DCS level in Jones et al., ${ }^{27}$ and the excellent quantitative agreement between the IAM-SCAR+I TCS and the SMCPP/BEB TCS. This latter observation is very encouraging, from the perspective of forming a comprehensive database for simulating electron transport in $\mathrm{pBQ}$.

\section{CONCLUSIONS}

We have reported on a series of experimental integral cross sections, for electron impact excitation $\left(E_{0}=20-40 \mathrm{eV}\right)$ of bands for composite vibrational quanta and bands of excited electronic-states in $\mathrm{pBQ}$. Those experimentally derived ICSs were supplemented with calculations using our IAM-SCAR+I model, our SMCPP model, and also a BEB method (with two different model chemistries) for the total ionization cross sections. Where a comparison between measurements and theory could be made, good quantitative (but in some cases only qualitative) agreement was typically found. Very good 
agreement between our elastic ICS from the SMCPP and IAMSCAR+I calculations is noted, with a similar accord between both theoretical results also being observed, over the common energy range, at the total cross section level. As one of the aims of this study was to provide absolute cross sections that could be incorporated into simulations of electron transport in $\mathrm{pBQ}$, we consider the present investigation to have been successful to some degree. Further work, however, even though pBQ is not a particularly nice species to work with experimentally, ${ }^{27-29}$ is needed to push the available measurements closer to threshold. An independent measurement of the TCS, in order to benchmark our IAM-SCAR+I and SMCPP/BEB TCS results, is also desirable. Furthermore, the effect of the continuum on our SMCPP computations should be explicitly determined. Nonetheless we believe that the present results and those from our earlier $\mathrm{pBQ}$ studies ${ }^{27-29}$ constitute a reasonable starting point, for electron transport simulations where pBQ is a constituent.

\section{ACKNOWLEDGMENTS}

This study was partially supported by the Australian Research Council (ARC) through Grant No. DP160120787. It was also partially supported by the Spanish Ministry MINECO (Project No. FIS2016-80440), the COST Action (No. CM301), and the ITN-Marie Curie (No. ARGENT-608163) European Union programme. M.A.P.L., M.T.do.N.V. (Grant I.D. No. 305672/2014-2), R.F.da.C., and M.H.F.B. all thank CNPq for financial support, while F.K. (Grant I.D. No. 2015/23792-5) also thanks FAPESP for financial support. Finally, M.H.F.B. acknowledges computational support from Professor Carlos de Carvalho at LFTC-DFis-UFPR and at LCPAD-UFPR and from CENAPAD-SP.

${ }^{1}$ Z. Lj. Petrović, S. Marjanović, S. Dujko, A. Banković, G. Malović, S. Buckman, G. García, R. White, and M. Brunger, Appl. Radiat. Isot. 83, 148 (2014).

${ }^{2}$ R. D. White, W. Tattersall, G. Boyle, R. E. Robson, S. Dujko, Z. Lj. Petrović, A. Banković, M. J. Brunger, J. P. Sullivan, S. J. Buckman, and G. García, Appl. Radiat. Isot. 83, 77 (2014).

${ }_{3}^{3}$ M. C. Fuss, A. G. Sanz, A. Muñoz, F. Blanco, M. J. Brunger, S. J. Buckman, P. Limão-Vieira, and G. García, Appl. Radiat. Isot. 83, 159 (2014).

${ }^{4}$ A. G. Sanz, M. C. Fuss, A. Muñoz, F. Blanco, P. Limão-Vieira, M. J. Brunger, S. J. Buckman, and G. García, Int. J. Radiat. Biol. 88, 71 (2012).

${ }^{5}$ M. J. Brunger, K. Ratnavelu, S. J. Buckman, D. B. Jones, A. Muñoz, F. Blanco, and G. García, Eur. Phys. J. D 70, 46 (2016).

${ }^{6}$ A. I. Lozano, K. Krupa, F. Ferreira da Silva, P. Limão-Vieira, F. Blanco, A. Munõz, D. B. Jones, M. J. Brunger, and G. García, Eur. Phys. J. D 71, 226 (2017).

${ }^{7}$ K. F. Ness, R. E. Robson, M. J. Brunger, and R. D. White, J. Chem. Phys. 136, 024318 (2012).

${ }^{8}$ J. de Urquijo, E. Basurto, A. M. Juárez, K. F. Ness, R. E. Robson, M. J. Brunger, and R. D. White, J. Chem. Phys. 141, 014308 (2014).

${ }^{9}$ H. V. Duque, T. P. T. Do, M. C. A. Lopes, D. A. Konovalov, R. D. White, M. J. Brunger, and D. B. Jones, J. Chem. Phys. 142, 124307 (2015).

${ }^{10}$ M. J. E. Casey, J. de Urquijo, L. N. Serkovic Loli, D. G. Cocks, G. J. Boyle, D. B. Jones, M. J. Brunger, and R. D. White, J. Chem. Phys. 147, 195103 (2017).

${ }^{11}$ P. J. Bruggeman, M. J. Kushner, B. R. Locke, J. G. E. Gardeniers, W. G. Graham, D. B. Graves et al., Plasma Sources Sci. Technol. 25, 053002 (2016).

${ }^{12}$ M. A. Ridenti, J. A. Filho, M. J. Brunger, R. F. da Costa, M. T. do N. Varella, M. H. F. Bettega, and M. A. P. Lima, Eur. Phys. J. D 70, 161 (2016).

${ }^{13}$ K. Bazaka, M. V. Jacob, and K. Ostrikov, Chem. Rev. 116, 163 (2016).
${ }^{14}$ L. Campbell and M. J. Brunger, Plasma Sources Sci. Technol. 22, 013002 (2013).

${ }^{15}$ P. Lavvas, R. V. Yelle, A. N. Heays, L. Campbell, M. J. Brunger, M. Galand, and V. Vuitton, Icarus 260, 29 (2015).

${ }^{16}$ L. Campbell and M. J. Brunger, Geophys. Res. Lett. 34, L22102, https://doi.org/10.1029/2007g1030442 (2007).

${ }^{17}$ L. Campbell and M. J. Brunger, Int. Rev. Phys. Chem. 35, 297 (2016).

${ }^{18}$ L. Campbell and M. J. Brunger, Plan. Space Sci. 151, 11 (2018).

${ }^{19}$ H. Tanaka, M. J. Brunger, L. Campbell, H. Kato, M. Hoshino, and A. R. P. Rau, Rev. Mod. Phys. 88, 025004 (2016).

${ }^{20}$ See http://sup-vamdc.vamdc.eu/ for an example of a comprehensive database.

${ }^{21}$ L. C. Pitchford, L. L. Alves, K. Bartschat, S. F. Biagi, M.-C. Bordage, I. Bray, C. E. Brion, M. J. Brunger, L. Campbell et al., Plasma Process. Polym. 14, 1600098 (2017).

${ }^{22}$ M. J. Brunger and S. J. Buckman, Phys. Rep. 357, 215 (2002).

${ }^{23}$ J.-S. Yoon, M.-Y. Song, H. Kato, M. Hoshino, H. Tanaka, M. J. Brunger, S. J. Buckman, and H. Cho, J. Phys. Chem. Ref. Data 39, 033106 (2010).

${ }^{24}$ K. Anzai, H. Kato, M. Hoshino, H. Tanaka, Y. Itikawa, L. Campbell, M. J. Brunger, S. J. Buckman, H. Cho, F. Blanco, G. García, P. Limão-Vieira, and O. Ingólfsson, Eur. Phys. J. D 66, 36 (2012).

${ }^{25}$ M. J. Brunger, Int. Rev. Phys. Chem. 36, 333 (2017).

${ }^{26}$ J. D. Gorfinkiel and S. Ptasinska, J. Phys. B: At., Mol. Opt. Phys. 50, 182001 (2017).

${ }^{27}$ D. B. Jones, P. Limão-Vieira, M. Mendes, N. C. Jones, S. V. Hoffman, R. F. da Costa, M. T. do N. Varella, M. H. F. Bettega, F. Blanco, G. García, O. Ingólfsson, M. A. P. Lima, and M. J. Brunger, J. Chem. Phys. 146, 184303 (2017).

${ }^{28}$ D. B. Jones, F. Blanco, G. García, R. F. da Costa, F. Kossoski, M. T. do N. Varella, M. H. F. Bettega, M. A. P. Lima, R. D. White, and M. J. Brunger, J. Chem. Phys. 147, 244304 (2017).

${ }^{29}$ D. B. Jones, R. F. da Costa, F. Kossoski, M. T. do N. Varella, M. H. F. Bettega, F. Ferreira da Silva, P. Limão-Vieira, G. García, M. A. P. Lima, R. D. White, and M. J. Brunger, J. Chem. Phys. 148, 124312 (2018).

${ }^{30}$ B. Huskinson, M. P. Marshak, C. Suh, S. Er, M. R. Gerhardt, C. J. Galvin, X. Chen, A. Aspuru-Guzik, R. G. Gordan, and M. J. Aziz, Nature 505, 195 (2014).

${ }^{31}$ Y. Ding and G. Yu, Angew. Chem., Int. Ed. 55, 4772 (2016).

${ }^{32}$ M. Allan, Chem. Phys. 84, 311 (1984).

${ }^{33}$ M. Allan, Chem. Phys. 81, 235 (1983).

${ }^{34}$ A. Loupas and J. D. Gorfinkiel, Phys. Chem. Chem. Phys. 19, 18252 (2017).

${ }^{35}$ R. F. C. Neves, D. B. Jones, M. C. A. Lopes, F. Blanco, G. García, K. Ratnavelu, and M. J. Brunger, J. Chem. Phys. 142, 194305 (2015)

${ }^{36}$ D. B. Jones, R. F. da Costa, M. T. do N. Varella, M. H. F. Bettega, M. A. P. Lima, F. Blanco, G. García, and M. J. Brunger, J. Chem. Phys. 144, 144303 (2016).

${ }^{37}$ M. J. Brunger and P. J. O. Teubner, Phys. Rev. A 41, 1413 (1990).

${ }^{38}$ E. N. Lassettre, J. Chem. Phys. 43, 4479 (1965).

${ }^{39}$ Z. Mašín, J. D. Gorfinkiel, D. B. Jones, S. M. Bellm, and M. J. Brunger, J. Chem. Phys. 136, 144310 (2012).

${ }^{40}$ R. F. da Costa, E. M. de Oliveira, M. H. F. Bettega, M. T. do N. Varella, D. B. Jones, M. J. Brunger, F. Blanco, R. Colmenares, P. LimãoVieira, G. García, and M. A. P. Lima, J. Chem. Phys. 142, 104304 (2015).

${ }^{41}$ L. Chiari, H. V. Duque, D. B. Jones, P. A. Thorn, Z. Pettifer, G. B. da Silva, P. Limão-Vieira, D. Duflot, M.-J. Hubin-Franskin, J. Delwiche, F. Blanco, G. García, M. C. A. Lopes, K. Ratnavelu, R. D. White, and M. J. Brunger, J. Chem. Phys. 141, 024301 (2014).

${ }^{42}$ M. C. Fuss, A. G. Sanz, F. Blanco, J. C. Oller, P. Limão-Vieira, M. J. Brunger, and G. García, Phys. Rev. A 88, 042702 (2013).

${ }^{43}$ F. Blanco and G. García, Chem. Phys. Lett. 635, 321 (2015).

${ }^{44}$ H. Kato, A. Suga, M. Hoshino, F. Blanco, G. García, P. Limão-Vieira, M. J. Brunger, and H. Tanaka, J. Chem. Phys. 136, 134313 (2012).

${ }^{45}$ J. R. Brunton, L. R. Hargreaves, T. M. Maddern, S. J. Buckman, G. García, F. Blanco, O. Zatsarinny, K. Bartschat, D. B. Jones, G. B. da Silva, and M. J. Brunger, J. Phys. B: At., Mol. Opt. Phys. 46, 245203 (2013).

${ }^{46}$ O. Zatsarinny, K. Bartschat, G. García, F. Blanco, L. R. Hargreaves, D. B. Jones, R. Murrie, J. R. Brunton, M. J. Brunger, M. Hoshino, and S. J. Buckman, Phys. Rev. A 83, 042702 (2011).

${ }^{47}$ Y.-K. Kim and M. E. Rudd, Phys. Rev. A 50, 3954 (1994).

${ }^{48}$ K. Takatsuka and V. McKoy, Phys. Rev. A 24, 2473 (1981); 30, 1734 (1984). 
${ }^{49}$ J. S. dos Santos, R. F. da Costa, and M. T. do N. Varella, J. Chem. Phys. 136, 084307 (2012).

${ }^{50}$ M. H. F. Bettega, L. G. Ferreira, and M. A. P. Lima, Phys. Rev. A 47, 1111 (1993).

${ }^{51}$ R. F. da Costa, F. J. da Paixão, and M. A. P. Lima, J. Phys. B: At., Mol. Opt. Phys. 37, L129 (2004).

${ }^{52}$ R. F. da Costa, M. T. do N. Varella, M. H. F. Bettega, and M. A. P. Lima, Eur. Phys. J. D 69, 159 (2015).
${ }^{53}$ W. Sun, M. A. Morrison, W. A. Isaacs, W. K. Trail, D. T. Alle, R. J. Gulley, M. J. Brennan, and S. J. Buckman, Phys. Rev. A 52, 1229 (1995).

${ }^{54}$ C. J. Noble, K. Higgins, G. Wöste, P. Duddy, P. G. Burke, P. J. O. Teubner, A. G. Middleton, and M. J. Brunger, Phys. Rev. Lett. 76, 3534 (1996).

${ }^{55}$ R. Curík and F. Gianturco, J. Phys. B: At., Mol. Opt. Phys. 35, 1235 (2002).

${ }^{56}$ R. Curík, P. Cársky, and M. Allan, J. Phys. B: At., Mol. Opt. Phys. 41, 115203 (2008). 